\title{
LA INVESTIGACIÓN EN EL ARTE -LA RELACIÓN ARTE Y CIENCIA, UNA INTRODUCCIÓN
}

\author{
H. Marcelo Zambrano U.
}

Fecha de recepción: abril 2016

Fecha de aceptación: mayo 2016

\begin{abstract}
Resumen
Generar conocimientos, no sólo es una práctica habitual de la ciencia, el arte con su construcción, ha ido aportando con sostenibles y sensibles ideas al desarrollo del ser humano. El arte y la ciencia han propiciado conocimientos desde espacios diferentes, estableciendo espacios de diálogo, y en otras indiferentes, con largas separaciones, creando límites que las alejaron una de otra. En la actualidad, se encuentran separadas sin conocimiento previo, cuando en un inicio salieron de una matriz: la techné griega. Si sus prácticas estuvieron altamente relacionadas, por qué hoy estas mismas prácticas las excluyen, las separan y las ubican en posiciones opuestas. Este texto expone las adversas condiciones a las que se han enfrentado y las coincidencias que las encuentran como antagonistas dentro de una misma historia.
\end{abstract}

Palabras clave: investigación artística, ciencia, arte, techné

\begin{abstract}
Develop knowledge, is a common practice in science, however art and its structure, has contributed to sustainable development and sensible ideas. Art and science have conducted knowledge from different spaces, creating discussion and boundaries from one another. Today, art and science are apart, although originally both had the same matrix: techné. If, in the beginning their practices were highly related, why today these practices exclude each other? This article shows the adverse conditions these elements have faced and shows art and science as antagonists within the same story.
\end{abstract}

Key words: artistic investigation, science, art, techné

\section{Autor:}

H. Marcelo Zambrano Unda (Quito, 1972). Docente en varias universidades. Magister en Estudios de la Cultura por la Universidad Andina Simón Bolívar, sede Quito y Diplomado en Teorías del Arte Contemporáneo por la Pontificia Universidad Javeriana, Bogotá, Colombia; Licenciado en Artes Plásticas por la Universidad Central del Ecuador; Técnico Superior en Diseño Gráfico por el Instituto Metropolitano de Diseńo, Quito. Actualmente interesado en la investigación sobre temas relacionados con arte contemporáneo, tecnología, educación y prácticas curatoriales. 


\section{Introducción}

La investigación artística o la investigación basada en arte, es un espacio relativamente nuevo de reflexión sobre y desde el ámbito artístico. Se encuentra enmarcada dentro de las fronteras académicas de las universidades y los centros de investigación, sin embargo, mantiene una pretendida independencia y autonomía, debido principalmente a su relación ambigua con el ámbito científico. Esta relación entre el arte y la ciencia, define algunas prácticas específicas de la investigación artística pero a la vez excluye otras, en tanto prácticas apócrifas, poco sistemáticas $y$, por ende, poco confiables. De todas formas, es el desarrollo de esta relación lo que define la importancia de la investigación artística. El presente texto introduce las características de esta relación e intenta establecer puntos desde los que sea posible reflexionar tanto sobre la misma relación entre arte y ciencia, como sobre la práctica de la investigación artística.

\section{Relación arte y ciencia}

En la antigua Grecia, no existía una palabra que defina al arte tal como se lo conoce actualmente. El arte estaba más cercano a la definición del antiguo término griego techné, que abarcaba toda actividad relacionada con las habilidades y las destrezas necesarias para la elaboración de algo. Habilidades y destrezas reconocidas como valores fundamentales en el acto de producción. Es decir, el concepto de "arte" en la antigüedad estaba enlazado en mayor medida con el acto de producción que con los productos elaborados resultantes.

Sin embargo, existía una ineludible condición adicional. Este acto de producción se encontraba determinado por el establecimiento de un conjunto de reglas definidas previamente (Tatarkiewicz, 2001, p.80). Planteaba una noción de "arte" como una práctica racional basada en un sistema de reglas generales que implicaba la constancia de un conocimiento previo que se encontraba enlazado con la razón, y se oponía, por tanto, a cualquier forma de actividad irracional relacionada con alguna manifestación estimulada por la inspiración o la intuición -elementos propios del acto de creación, diferente al de elaboración, como la poesía.

El antiguo término techné, incluía actividades diversas como hacer zapatos, construir embarcaciones, bordar, contar cuentos o cazar, pero además incluía actividades diferentes, pero no por ello alejadas de alguna forma de habilidad o destreza, como la medicina, la geometría, la gramática, la astronomía o la retórica. Posteriormente, después de la invasión romana al territorio griego aproximadamente en el siglo II a.C, el término techné -raíz de las palabras técnica y tecnología- se tradujo al latín como ars -raíz de la actual palabra arte.

Esta noción de "arte", o más específicamente "artes" en plural, fue la base sobre la que se fundamentaron las diversas clasificaciones de las artes en la antigüedad.

Las artes, entendidas como sistemas racionales de reglas o como un conjunto de métodos específicos dispuestos de manera sistemática, se organizaron en función de la inclusión de actividades que en la actualidad podrían denominarse como artesanales (oficios y actividades de carácter manual) y de otras actividades distintas que podrían relacionarse con lo que posteriormente se considerarían como ciencia. 
Las distintas clasificaciones de las artes catalogaron las diferentes disciplinas según criterios en extremo diversos. Así, se clasificaron según sus objetivos (placer o utilidad), según su relación con la realidad (objeto o imitación), según el tipo de esfuerzo realizado (artes liberales o artes vulgares), según sus productos resultantes (artes teóricas o artes prácticas), según su "valor" (artes mayores o artes menores), y según su nivel de espiritualidad (desde las artes que producen objetos físicos, hasta las artes puramente intelectuales) (Tatarkiewickz, 2001, p.85).

Sin embargo, no todas las clasificaciones perduraron a través del tiempo. Únicamente trascendieron las clasificaciones que catalogaron las disciplinas en función de la configuración jerárquica de la sociedad en la antigüedad, es decir, las que se encontraban definidas por sus objetivos -las artes que producían placer o las que tenían algún tipo de utilidad- y las que se encontraban definidas por el tipo de esfuerzo realizado -las artes liberales que podían ser practicadas por hombres libres, y las artes vulgares practicadas por el vulgo, las mujeres o los esclavos- clasificación basada en la aversión de los antiguos griegos al trabajo físico (Shiner, 2004, p.57).

La clasificación basada en el tipo de esfuerzo realizado dividía las disciplinas entre artes liberales y artes vulgares (o serviles), separaba las actividades netamente intelectuales, cultivadas por hombres libres, de las que se definían por la intervención de alguna forma de trabajo físico y/o pagado (Shiner, 2004, p.48). Las artes liberales estaban conformadas por la gramática, la retórica, la aritmética, la geometría, la astronomía, mientras las artes vulgares incluían la arquitectura, la escultura, la pintura y otras actividades de carácter manual.
En la Edad Media, esta clasificación se extendió y se definieron las fronteras entre disciplinas. Así, las artes liberales se dividieron en el trivium (gramática, retórica, lógica) y el quadrivium (aritmética, geometría, astronomía, música), mientras las artes vulgares (o serviles) pasaron a denominarse como mecánicas (tejido, armamento, comercio, agricultura, medicina, teatro, caza).

De esta forma, las disciplinas que actualmente reconocemos como artes (la pintura y la escultura) forman parte de la clasificación de las artes mecánicas debido a que se las consideraba como actividades inferiores (basadas en lo manual), mientras que las disciplinas que formaban el grupo de las artes liberales (el trivium y el quadrivium) se encontrarían dentro de lo que actualmente consideramos como disciplinas científicas.

Para el siglo XVI, un grupo de pintores y escultores junto a Giorgio Vasari fundaron una academia de dibujo con el objetivo de elevar a la pintura y a la escultura al estatus de artes liberales, argumentando que "sus miembros quedaban exentos de toda regla gremial" (Shiner, 2004, p.70). El propósito de este intento por posicionar a la pintura y a la escultura como actividades intelectuales (y significativamente fuera del ámbito reglado gremial) radicaba en la consideración de la imagen y posición del "artista" como trabajador intelectual, como practicante de una disciplina cercana a lo "científico".

En este sentido, la pintura y la escultura en tanto disciplinas cercanas a las artes mecánicas, requerían la adquisición y el aprendizaje de conocimientos como la geometría, la anatomía y la mitología, desplazando su práctica desde lo manual hacia lo intelectual, desde lo experiencial 
hacia lo racional, lo que permitió, por ende, la emergencia de la idea de una suerte de artista/ científico (Shiner, 2014, p.80) -Leonardo da Vinci y Alberto Durero, son ejemplos claros de esta figura.

Para el siglo XVIII, la división entre artes liberales y artes mecánicas resultaba cada vez menos útil para establecer fronteras y relaciones entre las diferentes disciplinas y ciencias. Si bien, aún se incluían dentro de la clasificación de las artes a la astronomía, las matemáticas y la geometría, otras disciplinas como la pintura, la escultura y la arquitectura, continuaban su lucha por la transformación de su estatus hacia el grupo de las artes liberales, es decir, hacia una definición más cercana de lo que actualmente se considera como científico.

Significativamente, este cambio de estatus se consolidó a partir de la constitución de varias instituciones relacionadas tanto con la determinación académica de la práctica artística como con la definición institucional de lo que actualmente se considera como ciencia. Así, la creación de la Academia de Dibujo en Florencia en 1563 y la Academia Romana de San Lucas en 1600 (Shiner, 2004, p.108), permitieron la consideración como artistas "libres" a sus miembros, mientras que la fundación de la Academie Francaise en 1635, determinó la distinción no sólo de la pintura, la escultura y la arquitectura, sino también de la poesía y las belles lettres, como artes liberales -según cita Larry Shiner a Antoine Furetiere, las letras humanas o "verdaderas belles lettres son la física, la geometría y las ciencias sólidas” (Shiner, 2004, p.110).

En un giro similar, las ciencias empiezan a definir sus límites y establecer sus espacios institucionales mediante la creación en 1663 de la Academie Royale des Sciences en Francia, y en
1760 la Royal Society en Londres (Shiner, 2004, p.111). Espacios que legitimaron las fronteras disciplinares y demarcaron a la ciencia como ámbito específico de conocimiento.

Esta estrecha relación entre el arte y la ciencia empieza a fragmentarse a partir del siglo XVIII. Las artes se separan de las prácticas artesanales, y las ciencias se separan de la clasificación de las artes. La configuración de las prácticas y disciplinas que permitían llamar a una actividad "arte" y suponía brindar un prestigio similar al que en la actualidad se determina como condición "científica", empieza a transformarse hacia nuevas formas de configuración.

Así, las artes -en la figura del artista/artesano como productor manual de cosas- se inclinaron por la definición de sus prácticas desde una configuración conformada por talento (ingenium) y genio (genius). Por un lado, el ingenium se relacionaba con el talento considerado como natural, como una predisposición propia hacia el ingenio y la imaginación. Por otro lado, el genius, en sus dos acepciones, significaba tanto guardián espiritual como ingenium o talento natural (Shiner, 2004, p.106).

No obstante, a pesar de que el artista debía poseer ingenium, debía impostergablemente cumplir las reglas establecidas a veces por el gremio de artistas/artesanos otras por las exigencias de la práctica que marcaba la experiencia. De esta manera, aunque el genius y la inspiración se consideraban como fundamentales en la práctica artística, no se ubicaban más allá de la razón y el juicio, por el contrario, la razón se articulaba directamente con el genio, la inspiración y la imaginación. Se explicaría, por tanto, la intención permanente de los pintores $y$ escultores por defender y demostrar que su actividad se enlazaba directamente con el intelecto y la razón. 
En este sentido, la práctica artística desarrollada en gran medida en talleres y caracterizada por el uso permanente del ingenium y la inspiración, desarrollaba su aprendizaje a través del ejemplo del oficio y la experimentación permanente, intentando no seguir modelos y recetas pero definiendo sus actividades a través de un conjunto estricto de reglas y regulaciones. De esta forma, empezaron a desarrollarse espacios de articulación entre los ámbitos de las artes liberales y las artes mecánicas, entre los ámbitos de lo que hoy consideraríamos como ciencia y lo que consideramos actualmente como arte.

Esta configuración particular de colaboración, "ofreció a los científicos un modelo de conocimiento basado en la experiencia y en el esfuerzo mancomunado" (Shiner, 2004, p.112), aprendido de la dinámica propia de los talleres en los que las artes mecánicas desarrollaban su práctica. En otras palabras, la unión de métodos experimentales y modelos matemáticos permitió que se sentaran las bases de lo que se conoce como ciencia.

Ahora bien, desde el momento en que las ciencias se separan de la clasificación de las artes y demarcan sus campos específicos de conocimiento, se definen como ámbitos autónomos e independientes, con características propias que difieren en gran medida de las particularidades de las artes.

Desde esta perspectiva, el objetivo último de la ciencia se define como el establecimiento de los parámetros con los que el ser humano obtiene los instrumentos necesarios para explicar de forma sistemática, controlable y adecuadamente sustentada el mundo en el que vive, y de esta forma entenderlo y conocer su posición en él (Nagel, 2006, p.20, 35). Los datos y la información con los que la ciencia explica los fenómenos del mundo se obtienen a través de procesos ordenados y sistemáticos agrupados en métodos denominados generalmente como investigación.

Según la UNESCO, la investigación se define como cualquier actividad sistemática emprendida con el propósito de incrementar la acumulación de conocimiento y el uso de estos conocimientos para descubrir nuevas aplicaciones (OECD, Glossary of Statistical Terms, 2008). Es decir, investigar incluye el estar consciente de que no se conoce, tener presente lo que no se conoce y lo que se desea conocer (Klein, 2010). Por tanto, todo proceso de investigación inicia con preguntas relevantes y emplea métodos apropiados que aseguren la validez y la confiabilidad de las respuestas a esas preguntas, documentando los resultados para posteriormente divulgarlos.

Estas características que exponen la práctica científica como altamente regulada y controlada permiten advertir las razones por las cuales ciertas disciplinas fueron consideradas en la antigüedad como parte de la clasificación de las artes. Para Aristóteles, las artes se definían como aquella predisposición a producir cosas, conocimiento, de un modo racional (Tatarkiewickz, 2001, p.80), es decir, fundamentaba esta predisposición en métodos, reglas y un orden preestablecido. Por tanto, a través de las artes y de sus métodos regulares y ordenados, también se podían comprender las cosas del mundo.

Ahora bien, en la actualidad estos dos ámbitos se mantienen separados. Por una parte, las ciencias se encuentran definidas por las reglas impuestas desde un orden dominado por la hegemonía de las Ciencias Naturales en la definición de la investigación como un conjunto de métodos altamente ordenados, regulados y controlados 
(Lesage, 2009), y por otra parte, el arte, que en sí mismo se define como una investigación empírica en tanto conjunto de métodos no sistemáticos (Borgdorff, 2009).

Sin embargo, desde cierta perspectiva y a través de ciertos procesos (como la investigación artística) las artes proponen nuevas formas para enlazar estos ámbitos separados, ámbitos que en algún momento se encontraron articulados de tal forma que resultaban casi indefinibles sus fronteras.

Para empezar, el arte es un ámbito que se ha configurado de forma distinta al ámbito de la ciencia. La enseñanza de la práctica artística se desarrolla en academias y universidades en las que la relación entre arte y ciencia se convierte en compleja. Si los artistas no están entrenados como los científicos, ni deberían ser entrenados como tales, desde la perspectiva hegemónica de las Ciencias Naturales señalada, la obligación de investigar en las academias y universidades no tendría sentido (Lesage, 2009)

Si bien, la investigación artística no busca producir conocimiento de la manera en la que lo hace la investigación científica, busca mejorar y ampliar el universo simbólico y estético en el que se desenvuelve el ser humano. En este sentido, la investigación artística incluye la producción de imágenes, narrativas, sonidos o experiencias, amplía la posibilidad de producción de sensaciones y sentidos.

En cuestiones metodológicas, la investigación artística toma distancia y se aleja de la tradicional sistematización metodológica científica debido a su acercamiento a procesos de búsqueda y de indagación no-sistemáticos y poco regulados, concediendo amplia importancia a los encuentros significativos (serendipia) que operan en los procesos de ensayo y error propios de la práctica artística.

En este punto, el arte y la academia, como forma institucional de la ciencia, según se señaló, sostienen una relación particular. Por un lado, lo académico se identifica como una etiqueta que representa calidad en las universidades, no obstante, lo académico también podría considerarse como sinónimo de pérdida de creatividad o pérdida de ingenio en la práctica artística. Así, el arte y la investigación artística juegan un rol importante en los procesos de "academización" de la práctica artística, como la búsqueda del desplazamiento del arte hacia una condición de hecho científico (por ejemplo en las llamadas clínicas artísticas, en la transformación del taller en "laboratorio" y en los constantes "mapeos" artísticos que se presumen datos a ser consumidos y utilizados en investigaciones científico-sociales) y en los procesos en los que la investigación científica se inclina hacia las exploraciones impredecibles y azarosas (Borgdorff, 2009), como en la crisis de un paradigma científico. Como los artistas, señala Kuhn, "los científicos creadores deben ser capaces de vivir, a veces, en un mundo desordenado (Kuhn, 1998, p.130).

En conclusión, esta relación entre ciencia y arte desemboca en un proceso denominado como investigación artística. Un proceso que se enmarca dentro de los límites de la práctica hegemónica de la investigación científica (sistematización, orden y control), manteniendo ciertas características propias del ámbito de las artes, como su necesidad de trabajo con el ingenium y la imaginación del talento natural, mientras se sostiene fija a un conjunto de reglas establecidas previamente. 
Según se ha desarrollado, desde hace mucho tiempo el arte y la ciencia han mantenido una relación compleja que se ha entrelazado y separado intermitentemente. Si el arte-que en la antigüedad contenía en sus clasificaciones a la ciencia en general- se entendía como "el recto ordenamiento de la razón" o "la recta idea de lo que debe producirse" (Tatarkiewicz, 2001, p.86), la ciencia actualmente debe entenderse como un espacio incluyente en el que el arte -a través de la investigación artística- provea nuevas formas de información con las cuales entender el mundo (Oxman, 2016).

Tener una actitud científica es remplazar sensaciones por conceptos, y expresar esos conceptos con palabras (Jacquard, 2005, p.21). Entonces, probablemente sea labor del arte proporcionar esas sensaciones.

\section{Referencias bibliográficas}

Borgdorff, H. (2009). Artistic Research within the Fields of Science. Sensuous Knowledge 6. Bergen: Bergen National Academy of the Arts.

Gray, C. and Malins, J. (1993). Research Procedures / Methodology for Artists \& Designers. Recuperado de http://www. carolegray.net/Papers\%20PDFs/epgad.pdf

Hannula, M. et al. (2005). Artistic Research. Theories, Methods and Practices. Helsinki: Academy of Fine Arts.

Hannula, M. (2009). Catch Me If You Can: Chances and Challenges of Artistic Research. Art \& Research. Recuperado de http://www. artandresearch.org.uk/v2n2/hannula1.html

Ito, J. (2016). Design and Science. MIT Press. Recuperado de http://jods.mitpress.mit.edu/ pub/designandscience

Jacquard, A. (2005). La ciencia para no científicos. México: Siglo XXI.

Klein, J. (2010). What is artistic research?. Research Catalogue. Recuperado de https:// www.researchcatalogue.net/view/15292/15293

Kuhn, T. S. (1992). La estructura de las revoluciones cientificas. México: Fondo de Cultura Económica.

Lesage, D. (2009). Who's Afraid of Artistic Research? On measuring artistic research output. Art \& Research. Recuperado de http://www. artandresearch.org.uk/v2n2/lesage.html

Nagel, L. (2006). La estructura de la ciencia. Barcelona: Paidós.

OECD. (2008). Glossary of Statistical Terms.

Oxman, N. (2016). Age of Entanglement. MIT Press. Recuperado de http://jods.mitpress.mit. edu/pub/AgeOfEntanglement

Shiner, L. (2004). La invención del arte. Una historia cultural. Barcelona: Paidós.

Tatarkiewicz, W. (2001). Historia de seis ideas. Arte, belleza, forma, creatividad, mimesis, experiencia estética. Madrid: Teknos. 